\title{
Closed-loop Controlled Brillouin Optical Time-Domain Analysis
}

\author{
Zhisheng Yang, Marcelo. A. Soto, Luc Thévenaz \\ EPFL, Swiss Federal Institute of Technology, Institute of Electrical Engineering, SCI-STI-LT Station \\ 11, CH-1015 Lausanne, zhisheng.yang@epfl.ch
}

\begin{abstract}
A closed-loop controlled BOTDA distributed optical fibre sensor is proposed for tracking fast temperature-strain evolution. The measurement time is reduced by two orders of magnitude with respect to classical BOTDA sensing, while keeping the same accuracy and measurement conditions.
\end{abstract}

\section{Introduction}

Brillouin optical time-domain analysis (BOTDA) can reliably monitor the distributed temperature and strain condition along long-distance optical fibre links ${ }^{1,2}$. In classical BOTDA, a pulsed pump wave amplifies a counter-propagating probe wave locally when the pump-probe frequency offset falls within the local Brillouin gain spectrum (BGS) of the sensing fibre. The spectral shape of the BGS is reconstructed by scanning the pumpprobe frequency offset, so that the peak gain frequency, designated as Brillouin frequency shift (BFS) $v_{B}$, can then be extracted at each fibre position by quadratic fitting ${ }^{1-3}$. The measurement time of BOTDA sensors in the $\mathrm{km}$-range is typically in the order of several minutes. This restricts classical BOTDA to measure quasistatic temperature and strain. The measurement process can be speeded up by reducing the frequency scanning time $e^{4}$ or fully eliminating the frequency scanning process using either a multifrequency comb $\mathrm{b}^{5}$ or a slope-assisted technique, thus requiring only one pump-probe frequency offset $^{6}$. Reducing the measurement time in these methods results in the degradation of other parameters, thus for instance leading to poorer spatial resolution ${ }^{5}$, larger BFS uncertainty ${ }^{4-6}$, or a limited temperature/strain measurement range ${ }^{6}$. In this paper, instead of using the above mentioned BOTDA methods based on 'openloop' protocols, we propose a novel closed-loop controlled BOTDA (CC-BOTDA) method for tracking the BFS evolution at each fibre position. Using this totally disruptive approach the BFS profile is directly obtained from the output of a closed-loop controller with no need of frequency scanning and post-processing. The proposed method offers the ability to track fully distributed temperature/strain changes in second scale, whilst providing a measurand resolution similar to classical BOTDA. In addition, the overall BFS spectral coverage can extend far beyond the BGS width. Results demonstrate distributed sensing along a $\sim 10 \mathrm{~km}$-long fibre, with $2 \mathrm{~m}$ spatial resolution, using a measurement time of $1.7 \mathrm{~s}$ (limited by our instrumentation) and reaching a BFS uncertainty of $0.23 \mathrm{MHz}$ with $256 \mathrm{x}$ single trace averaging. The measurement speed and BFS precision can be flexibly chosen by properly setting the parameters of the closedloop controller.

\section{Principle}

In the CC-BOTDA method an operation point at detuning $\Delta v_{o p}$ and gain amplitude $g_{o p}$ in the BGS is firstly defined, as shown in Fig. 1(a). The key concept of the approach is to track local BGS spectral shifts due to temperature or strain changes by compensating the local gain changes through a calculated modification of the pumpprobe frequency offset. A closed-loop (feedback) control system is used to correct any local gain amplitude change $\Delta g(z)$ resulting from local BFS variations. The predefined set-point $g_{o p}$ is tracked and held using a software PID control loop, so that the pump-probe offset distribution dynamically set by the controller actually represents the measured real time BFS distribution along the fibre.

From the implementation point of view, since the BFS distribution over the sensing fibre is not uniform, the sensing fibre of length $L$ has to be considered as a concatenation of $N$ short fibre segments of identical length $(N=L / \Delta z$, with $\Delta z$ the spatial resolution of the sensor), as shown in Fig. 1(b). The BFS in each fibre segments $v_{B}\left(z_{i}\right)$ has to be initially measured with moderate precision using a standard BOTDA interrogation.
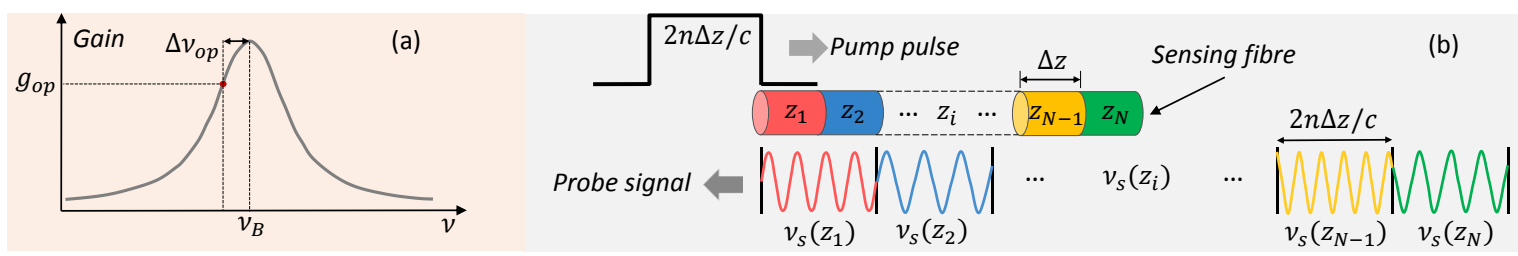

Fig. 1: (a) Operation point of the closed-loop system. (b) Schematic of the proposed method tailoring the probe frequency. 
The probe wave is then temporally sectioned in $N$ slots of same duration $\left(=2 N_{g} \Delta z / c\right.$, where $c / N_{g}$ is the group velocity). The probe frequency in each time slot is set according to the reference BFS profile, minus an offset $\Delta v_{o p}$. This way, when the pump pulse arrives at a given fibre position $z_{i}$, it will interact with the corresponding probe frequency matching the expected detuning $\Delta v_{o p}$. In a calibrated system, the probe is exactly detuned from peak gain by $\Delta v_{o p}$ at any position, so that the measured BOTDA trace shows a uniform exponential decay due to the pump attenuation, independently of the actual BFS profile, as depicted in the upper-left frame of Fig. 2. This figure shows the principle of the proposed CC-BOTDA. When a spectral shift of the local BGS occurs at time $t_{c}$, a local gain change $\Delta g\left(z_{i}\right)$ is induced with respect to the reference value $g_{\text {op }}$. The closed-loop controller corrects this change (see bottom frame in Fig. 2) by adjusting the probe frequency $\Delta v_{s}\left(z_{i}\right)$ in the corresponding time slot $z_{i}$. While the controller parameters define the speed and stability of the closed-loop system, updated with a delay $T_{d}$, the output of the controller $\Delta v_{s}\left(t_{c}+T_{d}, z_{i}\right)+\Delta v_{o p}$ corresponds to the distributed BFS profile along the fibre. In contrast to existing BOTDA schemes, in this case the BFS profile is not obtained by the usual BGS spectral fitting (as in standard BOTDA) or by direct conversion of gain amplitude variations (as in SABOTDA), but it is retrieved directly from the controller output signal.

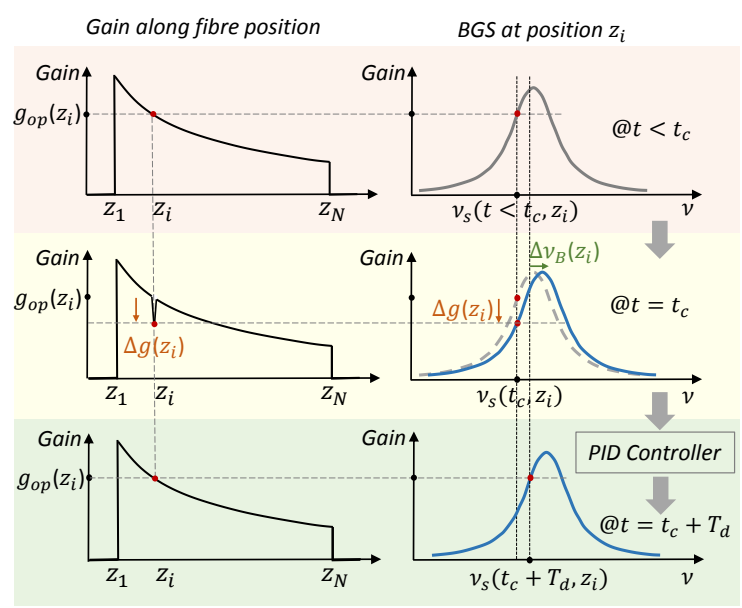

Fig. 2: Principle of the tracking.

\section{Experimental results and discussion}

In the experiment, a $\sim 9.3 \mathrm{~km}$-long sensing fibre is probed with a $2 \mathrm{~m}$ spatial resolution, thus defining 4650 time-domain slots on the probe wave that is classically generated by modulation using an arbitrary waveform generator (AWG). In order to achieve the highest sensitivity and minimise the impact of noise, the operating point $\Delta v_{o p}$ has been set at the maximum slope of the BGS
$\left(\Delta v_{o p}=F W H M / \sqrt{12}\right.$, equal to $17.3 \mathrm{MHz}$ for $2 \mathrm{~m}$ spatial resolution). Due to the $256 \mathrm{x}$ timeaveraging and the time required by the AWG to upload all frequency values in real-time, the sampling interval for the feedback loop (i.e. the tracking step interval) turns out to be $\sim 1.7 \mathrm{~s}$, which is essentially limited by the slow transfer speed to our AWG. By recording the probe frequencies for each tracking step, the BFS evolution as a function of time is obtained for all fibre positions at each trace acquisition.

As a proof-of-concept, a software proportionalintegrator $(\mathrm{PI})$ controller has been implemented in LabView, which directly transfers the PI output (probe frequency) to the AWG.

To optimise the proportional $\left(K_{P}\right)$ and integral $\left(K_{I}\right)$ feedback coefficients, the response of the closed-loop system has been measured while applying an instantaneous temperature step to a $6 \mathrm{~m}$-long fibre section. Starting from a reference BFS profile measured at $22^{\circ} \mathrm{C}$, and switching on the tracking system when the hotspot section is at $32^{\circ} \mathrm{C}$, the response to an equivalent temperature step of $10^{\circ} \mathrm{C}$ is measured. Following a standard procedure for the adjustment of a $\mathrm{PI}$ controller, $K_{P}$ is firstly adjusted while setting $K_{I}=0$. The response of the PI output is shown in Fig. 3(a). It can be seen that, when $K_{P}$ is set to the inverse of the BGS slope normalised by the local gain at the operation point $\left(K_{P}=33\right)$, the feedback loop can follow the $10^{\circ} \mathrm{C}$ temperature change in only one tracking step $(\sim 1.7 \mathrm{~s})$. However, this fast response leads to a relatively large temperature uncertainty $\left(1.61^{\circ} \mathrm{C}\right)$ at steady state, which is 5 times worse than the result obtained using classical BOTDA scanning $\left(\sim 0.24^{\circ} \mathrm{C}\right)$ with $1 \mathrm{MHz}$
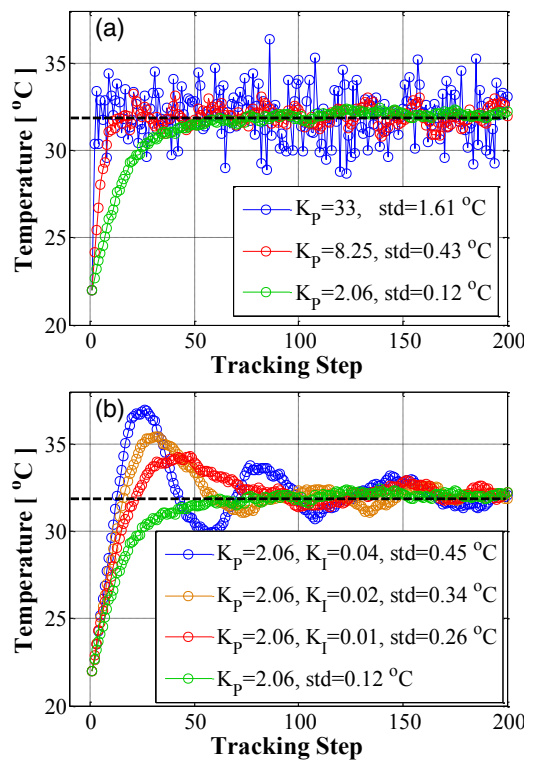

Fig. 3: Output of the tracking system for a $10^{\circ} \mathrm{C}$ temperature step using (a) only a proportional gain $K_{P}$, (b) PI controller with constant $K_{P}=2.06$ but different $K_{I}$. 
step and same number of averaging. Note that the uncertainty can be greatly reduced by decreasing the value of $K_{P}$, at the expense of a few more tracking steps. As exemplified by the green curve in Fig. 3(a), the uncertainty is reduced by a factor 13 with only 45 more tracking steps, while it needs $13^{2}=169$ times more averaged traces to reach the same uncertainty improvement in classical BOTDA. To even further increase the speed of the feedback loop, while keeping low noise level, a low $K_{P}$ must be used together with integration, as shown in Fig. 3(b). However, the higher the value of $K_{I}$, the higher are the overshoot and oscillations, thus increasing the temperature uncertainty. It can be observed that $K_{I}=0.01$ is a good choice, under which the temperature uncertainty is $0.26^{\circ} \mathrm{C}$ at $\sim 9.3 \mathrm{~km}$ distance. This is similar to the one obtained by classical BOTDA scanning $\left(\sim 0.24^{\circ} \mathrm{C}\right)$. Finally, to verify the tracking ability of the proposed CC-BOTDA system with large measurable BFS range, the temperature of the hotspot fibre section has been gradually increased from $22^{\circ} \mathrm{C}$ up to $53^{\circ} \mathrm{C}$, with a rate of $0.08^{\circ} \mathrm{C} / \mathrm{s}$ (i.e. $0.14^{\circ} \mathrm{C}$ per tracking step). Results obtained using different combination of $K_{P}$ and $K_{I}$ are shown in Fig. 4, where the blue curves represent the reference temperature evolution measured by a high-resolution electronic thermometer. One can observe that using $K_{P}=33$ (the inversed, normalised BGS slope), the system can follow the temperature evolution in quasi-real time, but with relatively large temperature uncertainty. As $K_{P}$ is reduced, the temperature uncertainty decreases linearly, but
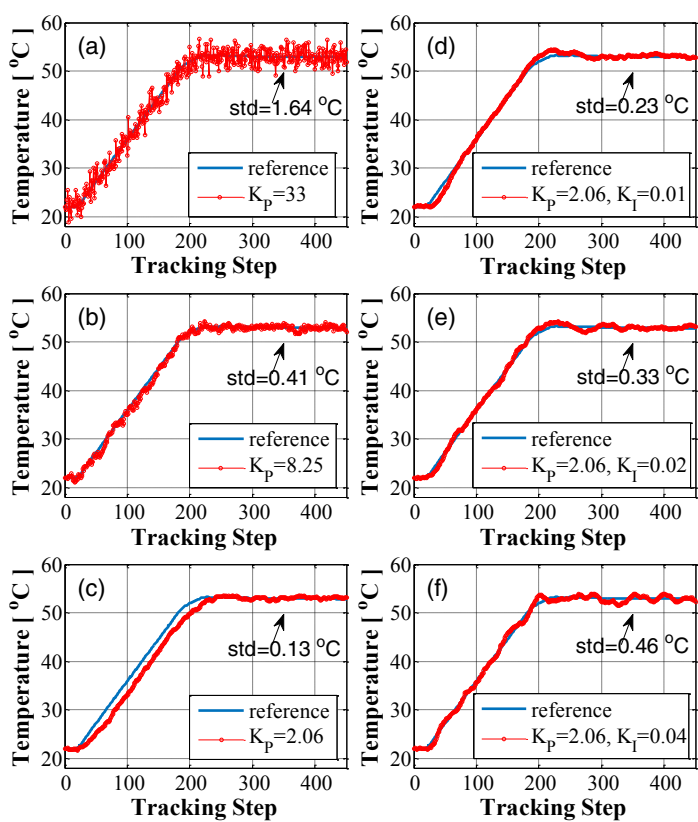

Fig. 4: Output of PI tracking system when applying a gradual temperature change at the rate of $0.08^{\circ} \mathrm{C} / \mathrm{s}$, using different $K_{P}$ and $K_{I}$ coefficients. at the cost of introducing a small delay. As shown in Fig. 4(c), when $K_{P}=2.06$, the delay turns out to be $\sim 15$ steps (i.e. $\sim 25 \mathrm{~s}$ ), while the temperature uncertainty is 2-fold better than standard BOTDA (which typically needs a few hundred scanning steps) with similar averaging. By introducing the integral term in low $K_{P}$ condition, the tracking speed can be increased, as shown in Fig. 4(d)(f). A negligible delay (tracking in one step, $1.7 \mathrm{~s}$ ) could be observed when setting $K_{I}=0.02$ or $K_{I}=0.04$, with a temperature precision similar to a classical BOTDA, demonstrating that the CCBOTDA can reduce the measurement time by two orders of magnitude with no penalty on accuracy. The correct tracking of the temperature over a $31^{\circ} \mathrm{C}$ range confirms that the method has conceptually an unlimited measurand range, not restricted to the BGS width.

\section{Conclusions}

A novel method is proposed and experimentally demonstrated to provide fast distributed temperature/strain sensing by optimally revisiting the procedure for collecting the relevant information. Since by concept the method totally suppresses the necessity of a spectral scanning, the measurement time is drastically reduced when compared to standard BOTDAs. The time required to reach a correct (steady-state) measurement is fixed by the closed-loop bandwidth, practically limited by the instrumental time required to upload the probe frequency sequence. A trade-off between acquisition speed and BFS uncertainty is present in linear proportion, in contrast with the square dependence resulting from time averaging.

The authors acknowledge the support from the Swiss Commission for Technology and Innovation (Project 18337.2 PFNM-NM).

\section{References}

[1] T. Horiguchi et al., "Development of a distributed sensing technique using Brillouin scattering," J. Lightwave Technol., Vol. 13, no. 7, p. 1296 (1995).

[2] A. Motil, et al., "State of the art of Brillouin fiber optic distributed sensing," Optics \& Laser Technology, Vol. 78, part A, p. 81 (2016).

[3] M. A. Soto et al., "Modeling and evaluating the performance of Brillouin distributed optical fiber sensors," Opt. Express, Vol. 21, no. 25, p. 31347 (2013).

[4] Y. Peled et al., "Fast Brillouin optical time domain analysis for dynamic sensing," Opt. Express, Vol. 20, no. 8, p. 8584 (2012).

[5] A. Voskoboinik et al., "Sweeping-free distributed Brillouin time-domain analyser (SF-BOTDA)," Opt. Express, Vol. 19, no. 26, p. B842 (2011).

[6] Y. Peled, et al., "Slope-assisted fast distributed sensing in optical fibers with arbitrary Brillouin profile," Opt. Express, Vol. 19, no. 21, p. 19845 (2011). 